\title{
Multicriteria optimization model to generate on-DEM Optimal Channel Networks
}

Simone Bizzi ${ }^{1}$,Andrea Cominola ${ }^{1,2}$, Emanuele Mason ${ }^{1}$, Andrea Castelletti ${ }^{1,3}$,

Kyungrock Paik ${ }^{4}$

$1+\frac{1}{r}$

Kyungrock Paik, paik@korea.ac.kr

${ }^{1}$ Department of Electronics, Information, and Bioengineering, Politecnico di Milano,

Piazza Leonardo da Vinci, 32, 20133

Milano, Italy

${ }^{2}$ Chair of Fluid System Dynamics,

Technische Universität Berlin, Straße des

17. Juni 135, 10623 Berlin, Germany

${ }^{3}$ Department of Civil and Environmental

Engineering, Swiss Federal Institute of

Technology, Stefano-Franscini-Platz,5, 8093

Zurich, Switzerland

${ }^{4}$ School of Civil, Environmental, and

Architectural Engineering, Korea

This article has been accepted for publication and undergone full peer review but has not been through the copyediting, typesetting, pagination and proofreading process, which may lead to differences between this version and the Version of Record. Please cite this article as doi: 10.1029/2018WR022977

(C)2018 American Geophysical Union. All Rights Reserved. 
Abstract. The theory of Optimal Channel Networks (OCNs) explains the existence of self-similarities in river networks by multiple optimality principles, namely i) the minimum energy expenditure in any link, ii) the equal energy expenditure per unit area of channel anywhere, and iii) the minimum Total Energy Expenditure (TEE). These principles have been used to generate OCNs from 2D networks. The existing notion of OCN considers the concavity of river longitudinal profiles as a-priori condition. Attempts to generate OCNs starting from a random 3D Digital Elevation Model (DEM) and minimizing solely TEE have failed to reproduce concave profiles. Yet, alternative approaches can be devised from the three optimality principles, for instance focusing on the local energy expenditure (LEE). In this paper, we propose a Multi-objective modeling framework for Riverscape Exploration (MoRE) via simultaneous optimization of multiple OCN criteria. MoRE adopts a multi-objective evolutionary algorithm and radial basis functions to efficiently guide DEM elevation variations required to shape 3D OCNs. By minimizing both TEE and the variance in LEE, MoRE successfully reproduces realistic on-DEM, OCN-based riverscapes, for the first time. Simulated networks possess scaling laws of upstream area and length, and river longitudinal profile resembling those of real river networks. The profile concavity

University, 145 Anam-ro, Seongbuk-gu,

Seoul 02841, South Korea

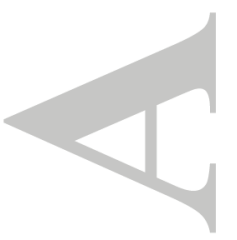

(C)2018 American Geophysical Union. All Rights Reserved. 
of generated on-DEM OCNs emerges as a consequence of the minimization of TEE constrained to the equalization of LEE. Minimizing TEE under this condition generates networks that possess specific patterns of LEE, where the scaling of slope with basin area resembles the patterns observed in real river networks.

\section{Keypoints:}

- On-DEM Optimal Channel Networks are generated via a multi-objective framework adopting multiple optimality principles

- The generated on-DEM Optimal Channel Networks possess the topological features and concavity of real river networks

- Trade-offs exist between topographic and topological efficiencies along the search for OCNs

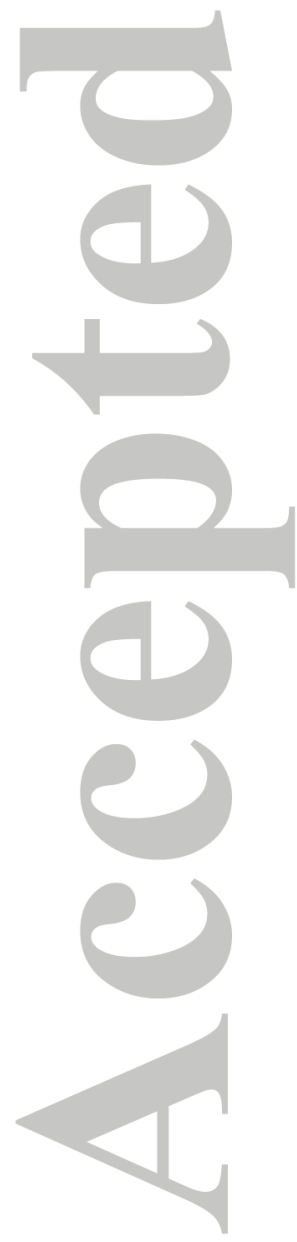




\section{Introduction}

Drainage networks are shaped through the complex interplay among various climatic, geomorphic, hydrologic, and tectonic processes that act across multiple spatio-temporal scales. The combined action of these processes creates the variety of river network configurations found in nature [Brierley et al., 2013]. Despite diverse generative processes and different dominant controls across the Earth, naturally formed river networks exhibit common features, such as fractal (self-similar) tree organization and concave longitudinal channel profiles [Horton, 1945; Flint, 1974; Rinaldo et al., 1998; Dodds and Rothman, 2000]. Water and sediment fluxes interact at the local scale, and lead to scale-invariant hydro-morphological features at the landscape scale. A fundamental question is whether the emergence of such organizational features in fluvial landscapes is driven by any kind of natural necessity, and how the physical interactions of water and sediment fluxes give shape to this necessity, ultimately creating organized structures across a basin. The mechanistic principle to explain the formation of such ubiquity has been a key quest [Kirkby, 1971; Rodriguez-Iturbe et al., 1992b; Paik and Kumar, 2010; Rinaldo et al., 2014]. An interesting interpretation in the literature suggests that fractal features in river networks are generated by their tendency to develop optimal connectivity paths. Through its erosive action, water carves channels and, subsequently, networks in a way to minimize the dissipation of the energy or work associated with its flow [Woldenberg, 1969; Kirkby, 1971]. This idea originated the concept of Optimal Channel Networks (OCNs) [RodriguezIturbe et al., 1992b; Rinaldo et al., 2014]. OCN theory explains how to reproduce the scale-invariant features that characterize river networks, despite their intrinsic dynamics.

(C)2018 American Geophysical Union. All Rights Reserved. 
These features hold statistically for most river basins worldwide, under the assumption that some sort of equilibria are reached by the river systems within the landscape. Here, we consider an OCN as a river network at the dynamic equilibrium (also called steady state), where mass uplift by tectonic processes is balanced by the erosive actions [Hack, 1960; Willgoose, 1994].

OCN configurations are identified by minimizing the criterion of the Total Energy Expenditure (TEE) [Rodriguez-Iturbe et al., 1992b]:

$$
\mathrm{TEE}=\psi \sum_{i=1}^{n} Q_{i}^{a} L_{i},
$$

where $Q_{i}$ and $L_{i}$ are discharge and length of the $i$-th reach, respectively, $a$ is a scaling exponent that typically ranges from 0.4 to 0.6 , and $\psi$ is a constant that depends on channel geometry, flow resistance, and properties of soil and liquid. The above formulation was defined for an arbitrary network composed of $n$ flow reaches [Rodriguez-Iturbe et al., 1992b], yet it also holds for an entire landscape where multiple river networks are located. In this latter case, $n$ refers to the total number of reaches in the domain [Sun et al., 1994]. This formulation of the criterion was derived by Rodriguez-Iturbe et al. [1992b] from a general expression of the work required to distribute flows of water and sediments along a drainage network. Three optimality principles are postulated to simplify the general expression to the TEE criterion, namely i) the minimum energy expenditure in any link of the network, ii) the equal energy expenditure per unit area of channel anywhere in the network, and iii) the minimum energy expenditure in the network as a whole [RodriguezIturbe et al., 1992b].

2D channel networks with minimum TEE yield tree statistics comparable to those of real river networks [Rodriguez-Iturbe et al., 1992b; Rinaldo et al., 1992]. This is a necessary, (C)2018 American Geophysical Union. All Rights Reserved. 
yet not sufficient, condition to claim that minimum TEE is the general signature of river network structures [Paik, 2012]. 2D network configurations are the planar projection of the underlying 3D landscapes. If an optimality signature of the riverscape structure is known, it should capture the 3D features of the landscape, not only the 2D characteristics of river networks. In this regard, an important feature is represented by the slope-area relationship [Flint, 1974; Willgoose, 1994]. Along the longitudinal profile of a river, its

local slope $S_{i}$ and upslope area $A_{i}$ are found to be related through a power-law relationship of the form

$$
S_{i} \propto A_{i}^{-\theta}
$$

where the concavity index $\theta$ has been found to be positive in most river networks, implying a concave upward longitudinal profile of rivers [Kirby and Whipple, 2012]. The seminal OCN study [Rodriguez-Iturbe et al., 1992b] derives an alternative criterion from the three principles of optimality, which highlights the principle of equal energy expenditure per unit area of channel anywhere in the network. We call this criterion equal Local Energy Expenditure (LEE), formulated as $Q_{i}^{a} S_{i}=$ const. This formulation is closely related to Eq. (2), and similarly describes the concavity of river longitudinal profiles. Indeed, Eq. (1) can be derived using the slope-area relationship [Rinaldo et al., 1996], where $\theta=1-a$. It has also been shown that a landscape complying with the slope-area power-law yields a constant energy expenditure, whose sum is postulated to be minimum [Sinclair and Ball, 1996]. Yet, all these studies assume the slope-area relationship as $a$ priori condition for generating OCNs. Indeed, this a-priori condition can be practically used a-posteriori to derive the elevation of $2 \mathrm{D}$ channel networks generated via the original

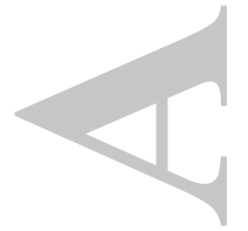

(C)2018 American Geophysical Union. All Rights Reserved. 
OCN algorithm [Abed-Elmdoust et al., 2016], because TEE depends only on the planar network organization, i.e., discharge distribution and reach length.

The OCN theory has been also applied to 3D landscapes, where Digital Elevation Models (DEMs) are altered to generate alternative channel network configurations (here named on-DEM OCNs). A synthetic experiment where an arbitrary 3D landscape with uniform slope is modified to meet the minimum TEE criterion alone failed to reproduce river profile concavity $[$ Paik, 2011]. The reason behind this failure has been revealed analytically [Paik, 2012] and is rather straightforward: equal local energy expenditure cannot be imposed a-priori in on-DEM OCNs, because altering the DEM topography influences both the planar network organization, which is measured by the TEE criterion, and the distribution of contributing areas with respect to channel elevation and slopes, which is instead captured by the LEE criterion. Paik [2012] also demonstrated that none of the optimal criteria proposed in literature (TEE, total stream power, and total energy dissipation rate) are capable individually of generating on-DEM OCNs having characteristics of real river networks. When constructing on-DEM OCNs, neither TEE nor LEE alone capture the pivotal link between the second and the third principles of optimality, namely the local energy expenditure regardless of the network topological structure, and the optimal arrangements of its elements, as implied by the principle of energy expenditure in the network as a whole. Conversely, this pivotal link must be included in the optimization problem to properly transfer the OCN theory to a 3D domain.

We suggest that a single criterion cannot properly explain the multiple necessities and factors that affect the development of river networks at the basin scale, in a 3D environment. This is in line with the original OCN theory, which postulated three different (C)2018 American Geophysical Union. All Rights Reserved. 
principles to describe how energy is distributed locally and globally across a basin, and its links. In this paper, we aim to demonstrate for the first time that it is possible to generate on-DEM OCNs, which simultaneously respect all the three hypotheses proposed in the OCN theory by Rodriguez-Iturbe et al. [1992b]. The objective of this study is to test whether river networks generated on a 3D landscape and conditioned by multiple OCN criteria exhibit realistic 3D river characteristics (i.e., network organization and longitudinal channel profile concavity), statistically comparable to those of real channel networks. This would allow for the first time to generate 3D OCN purely from optimality principles without imposing any slope-area relationship since this feature emerges as a consequence of the simultaneous application of OCN principles.

In pursuing the above objective, we develop the Multi-objective Riverscape Exploration (MoRE) modelling framework. Starting from a DEM, MoRE uses an evolutionary algorithm to search for alternative configurations of DEM cell elevations that lead to efficient channel networks, according to multiple OCN criteria. DEM cell elevation variations are expressed as a function of local hydrological and topographical conditions, implemented via Radial Basis Functions to reduce the complexity of the optimization problem. MoRE enables then the exploration of alternative on-DEM OCNs, whose basin features are then compared in terms of scaling laws of upstream drainage area, upstream length and slopearea relationship, with those observed in real river networks.

\section{Methods}

\subsection{On-DEM OCN problem formulation and experimental settings}

The original OCN theory [Rodriguez-Iturbe et al., 1992b] has its core in the following three principles: i) minimum energy expenditure in any link of the network; ii) equal (C)2018 American Geophysical Union. All Rights Reserved. 
energy expenditure per unit area of channel anywhere in the network; iii) minimum total energy expenditure in the network as a whole.

The first principle expresses a local optimal condition on channel geometry and results in an analytical solution of $H \propto Q^{f}$ with the exponent $f=0.5$, where $H$ is the local hydraulic depth of a river channel and $Q$ its discharge [Rodriguez-Iturbe et al., 1992b]. This solution is considered to be close to that of observed at-a-station hydraulic geometry relationships $(f \approx 0.4)$ [Leopold and Maddock, 1953]. We follow this approximation in this study, which leads to $a=0.5$ in Eq. (1) [Rodriguez-Iturbe et al., 1992b]. Moreover, under this principle, any link in the network spends the least energy to carry the discharge, and so it is not necessary to include detailed features, e.g., hydraulic depth, in our model. Instead, we adopt the discretized domain of a DEM composed of discrete unit cells, where each cell contains a river reach and its position is determined by its $3 \mathrm{D}$ coordinates. Given this setting, we prefer using the word "riverscape" in this work, rather than "landscape". Considering the second and third OCN principles, we formulate the problem of searching for on-DEM OCNs as alternative network configurations generated by following the criterion of minimum TEE, equal LEE, or any combination of these two. In fact, multiple river network configurations can be found to have minimum TEE, meaning that the minimization problem has potentially multiple local minima. Among them, LEE varies according to the degree of the network branching [Rodriguez-Iturbe et al., 1992b]: each alternative configuration constitutes a different trade-off of the two criteria. By identifying these alternative trade-offs, we avoid the need of a-priori imposing the slope-area relationship: we directly produce different local elevation variations required to generate alternative OCNs over an unconstrained 3D topography.

(C)2018 American Geophysical Union. All Rights Reserved. 
To implement this idea, we develop the Multi-objective Riverscape Exploration modeling framework (MoRE). We use MoRE to examine the coupled effect of minimum TEE and equal LEE on riverscape formation. Multi-objective analytic approaches are generally used to explicitly evaluate conflicting objectives and produce a set of equally optimal solutions (i.e., Pareto-optimal). In MoRE, we include minimum TEE as the first objective, formulated as Eq. (1) ( $i$ now refers to the DEM cells, and $a=0.5$, as explained above). The second principle assumes a constant slope-area power-law. In the problem formulation we implement this objective in its statistical meaning: indeed, it has been shown that LEE holds statistically on a landscape. This means that the expected values of the slope-area relationship is constant across the basin [Tarboton et al., 1991; Rinaldo et al., 1992]. For this reason the second objective is formulated as the minimization of the variance of LEE (VarLEE), i.e., calculated as:

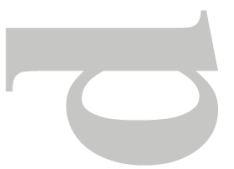

$$
\operatorname{VarLEE}=\operatorname{Var}\left[Q_{i}^{a} S\right]
$$

We approximate the discharge $Q_{i}$ in Eq. (1) and (3) with the upslope area $A_{i}$, assuming rainfall is uniform over the area [Paik, 2011]. No metric units need to be assigned in our theoretical study and hence the area $A_{i}$ at cell $i$ is simply equal to the number of upstream cells.

We adopt an initial riverscape similar to that considered by Paik [2011], i.e., a pyramidal DEM with a square base area of $100 \times 100$ unit cells (see as an example Figure 1 ). The DEM boundary cells have zero altitude, and each side of the pyramid has a uniform slope of 10 altitude units rise per cell in the upslope direction. This results in a peak altitude of 490 units. We then add a Gaussian noise in elevation with zero mean and $2 \sqrt{2}$ standard deviation to this ideal surface, to introduce variations in elevation and gradient and, thus, (C)2018 American Geophysical Union. All Rights Reserved. 
randomized flow directions in the initial DEM, represented as A1 in the top of Figure 1. For a raster DEM, the diagonal flow length is $\sqrt{2}$ times greater than the corresponding cardinal flow length. In contrast, other types of discretization can yield equal flow length in diagonal and cardinal directions. Considering that TEE formulation (1) includes flow length, the DEM discretization method may affect optimization results [Paik, 2012]. In this research, we evaluated the impact of the discretization method by comparing cases of larger cell diagonal length and uniform flow length, and found that the influence is insignificant and does not affect any conclusions of this study.

We assume that the the size of each DEM cell is larger than the support area needed to support the formation of channels [Tarboton et al., 1989; Schumm, 1956]. Under this assumption, hillslope processes are treated as embedded at the subgrid level and the modeling results can be interpreted solely as channel networks, consistent with the OCN concept.

Starting from the above initial configuration, MoRE explores the set of possible DEMs via optimization, allowing each cell to vary its altitude in the range of \pm 20 units. We chose this range as a reasonable compromise between the two needs of reducing the computational burden and allowing enough elevation difference between nearby cells to force changes in local flow directions.

By adopting this approach, we aim to investigate the relationship between network efficiency (TEE, third principle) and energy distribution across the landscape (LEE, second principle), which defines basin morphology and, ultimately, channel profiles (concavity). Starting from a theoretical topography represented by a DEM, we perturb the elevation of individual cells and explore how water and sediment are redistributed in order to mini(C)2018 American Geophysical Union. All Rights Reserved. 
mize TEE and VarLEE. We then examine connections between the two criteria, i.e., how basin topography (represented by LEE) needs to be adjusted to accommodate more efficient networks in terms of TEE. Eventually, we investigate whether the modeled on-DEM OCNs possess statistical tree properties and channel profile concavities comparable to those observed in real river networks.

\subsection{MoRE framework}

MoRE solves the following multi-objective problem:

$$
\min \text { [TEE, VarLEE] }
$$

The constant $\psi$ in Eq. (1) does not affect the optimization, thus all TEE values in this work are effectively normalized with respect to $\psi$. Moreover, the above problem can be better formulated as a function of the vector of decision variables $\mathbf{v}$, which should represent the elevations of DEM cells or a proxy for them, as we later explain:

$$
\min _{\mathbf{v} \in V} F(\mathbf{v})=\min _{\mathbf{v} \in V}[\operatorname{TEE}(\cdot ; \mathbf{v}), \operatorname{VarLEE}(\cdot ; \mathbf{v})]
$$

The decision variables in $\mathbf{v}$ belong to the decision space $V: Y$ real-valued decision variables are considered here, hence $\mathbf{v}=\left(v_{1}, v_{2}, \ldots, v_{Y}\right) \in \mathbb{R}^{Y}$. A direct approach to solve the minimization problem (5) would consider the elevation of each cell in a DEM as a decision variable in v. Yet, the search space, i.e., the size of possible DEMs, dramatically grows with the number of cells (or reaches) $n$ and the range of elevation change of each DEM cell per iteration. For example, if a DEM is composed of $100 \times 100$ cells and each cell can vary by up to 20 discrete elevation (vertical) units, $20^{10^{4}}$ alternative configurations could be evaluated. If we consider continuous elevation alterations, then an enumeration of the possible configurations is not even possible. In fact, several configurations are physically

(C)2018 American Geophysical Union. All Rights Reserved. 
nonsensical, and their exploration is worthless. In order to reduce the search space to only meaningful DEMs, we introduce a functional controller to guide the search toward elevation alterations that lead to more efficient channel networks.

We model the elevation change as a function of local upslope area and slope at cell level, namely $\left(z_{i}^{1}-z_{i}^{0}\right)=f\left(A_{i}, S_{i}\right)$, where $z_{i}^{0}$ and $z_{i}^{1}$ are the elevations of cell $i$ in the initial and altered DEM, respectively. Thus, the functional controller $(f(\cdot))$ allows to represent the variation in elevation as a function of local hydrological and topographical conditions. The arguments of the functional $f(\cdot)$ reflect the formulation of a previous genetic landscape evolution model [Paik, 2011]. However, no commonly accepted mathematical form for the functional itself was found in the literature, as the proposed formulations all have undetermined parameters. To avoid introducing limiting assumptions in MoRE, we mathematically express the $f(\cdot)$ relation via Radial Basis Functions (RBFs) [Park and Sandberg, 1991]. RBFs are a class of universal approximator functions, which approximate an output with a linear combination of real-valued radially symmetric activation functions, whose values depend on the distance from the RBF center.

We empirically select the number of RBFs in order to balance approximation capabilities and number of parameters, the latter reflecting into the number of dimensions $Y$ of the search space $V$. The configuration we selected is characterized by three Gaussian basis functions and assumes the form of Eq. (6) and (7):

$$
\left(z_{i}^{1}-z_{i}^{0}\right)=\sum_{k=1}^{K} w_{k} \operatorname{RBF}_{k}(\chi ; \mathbf{v})
$$

where $K$ is the number of basis functions $\operatorname{RBF}_{k}(\chi ; \mathbf{v}), \chi_{i}$ is the vector of inputs for cell $i$, and $w_{k}$ the weight of the $k$-th RBF within the network. The weights are formulated such that they are non-negative (i.e., $w_{k} \geq 0, \forall k$ ), and convex (i.e., $\sum_{k=1}^{K} w_{k}=1$ ). The single (C)2018 American Geophysical Union. All Rights Reserved. 
basis function is defined as follows,

$$
\mathrm{RBF}_{k}\left(\chi_{i}\right)=\exp \left[-\sum_{j=1}^{2} \frac{\left(\chi_{i, j}-c_{j, k}\right)^{2}}{b_{j, k}^{2}}\right]
$$

where $c_{j, k}$ and $b_{j, k}$ are the center and radius in the $j$-dimension of the $k$-th $\mathrm{RBF}$, respec-

tively.

In this study, $\chi_{i}$ contains information on cell $i$ of the starting DEM, with its elevation $z^{0}$, and is either the upslope area for $j=1$, or a smoothened slope value for $j=2$, the latter obtained by applying a moving average filter that spans $5 \times 5$ nearest-neighbour cells. The smoothing is only applied when composing the RBF inputs because Gaussian RBFs work best on continuously varying inputs, whereas raw slope values result in very high variability. In addition, both values of drainage area and slope vary linearly between their minimum and maximum values before composing the $\chi$ vector. This operation bounds the center and radius of all RBFs (i.e., using normalized variables, $c_{j, k} \in[-1,1]$ and $\left.b_{j, k} \in(0,1]\right)$. Therefore, the dimension of the parameter vector, i.e., the decision variables in our problem, is $Y=5 \times K=15$ for $K=3$, and each parameter has a lower and upper bound. Elevation alteration $\left(z_{i}^{1}-z_{i}^{0}\right)$ is finally obtained by uniformly rescaling back the RBF output between two reference values, i.e., \pm 20 units. As these lower and upper bounds limit the variation of each cell elevation, they locally represent the constraint derived by investigating landscapes at the steady state or dynamic equilibrium, where the mass gained by uplift balances the loss of sediments from the whole landscape [Paik, $2012]$

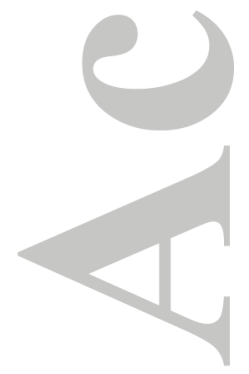

(C)2018 American Geophysical Union. All Rights Reserved. 
The minimization problem is completely defined by the set of equations below, where superscripts 0 and 1 refer, respectively, to starting and altered DEMs:

$$
\begin{gathered}
\qquad \mathbf{v}^{*}=\arg \min _{\mathbf{v}}[\operatorname{TEE}(A, L ; \mathbf{v}), \operatorname{VarLEE}(A, S ; \mathbf{v})] \\
A^{1}=f\left(z^{1}\right) ; \quad L^{1}=f\left(z^{1}\right) ; \quad S^{1}=f\left(z^{1}\right) \\
\text { subject to } \quad z^{1}=z^{0}+\sum_{k=1}^{K} w_{k} \operatorname{RBF}_{k}\left(\sqrt{A^{0}}, S^{0} ; \mathbf{v}\right)
\end{gathered}
$$

Eqs. $(1,3,6,7)$

It is worth noting that the information used to evaluate the altitude alteration refers to the starting DEM $z^{0}$ while the information used to evaluate the objectives is based on the new, altered DEM $z^{1}$. With the above formulation, the minimization problem is thus simplified into the search for the optimal RBF parameterizations. Due to the existence of alternative trade-offs between TEE and VarLEE, multiple solutions exist for Eq. (8), each consisting of a different DEM and its corresponding OCN.

In MoRE, we adopt Multi-Objective Evolutionary Algorithms (MOEAs) to solve Eq. (8) as they can tackle complexity and nonlinearities. MOEAs are iterative search algorithms that generate a Pareto-approximate set of solutions by mimicking the randomized mating, selection, and mutation operations that occur in natural populations [Coello et al., 2002]. These mechanisms allow MOEAs to deal with the challenges typically posed by multi-objective optimization problems, i.e., multi-modality, nonlinearity, and discreteness. Among the state-of-the-art MOEAs, we selected the Borg Multi-Objective Evolutionary Algorithm (Borg MOEA; Hadka and Reed [2013]). Its adaptive operator recombination and randomized restarts generate DEM configurations avoiding both local optima and strong dependency on the initial population settings. Since the optimization procedure is based on heuristics, an unbiasing procedure is adopted to filter its randomness: following (C)2018 American Geophysical Union. All Rights Reserved. 
a Monte Carlo approach, we repeated each optimization 10 times with different seeds for the random number generator. This number of seeds is selected to balance computational effort and results robustness.

The Borg MOEA enables robust optimization by assimilating components from other benchmark MOEAs, but it does not always result in an optimal solution [Hadka and Reed, 2012]. As a benchmarking test, we compared the results of MoRE against solutions randomly sampled from the search space, which serve as a baseline reference. Unbiased sampling is performed according to the method of Saltelli et al. [2010], which combines uniform sampling between lower and upper bound of each RBF parameter in statistically independent tuples. We relied on the SALib python library ${ }^{1}$, and evaluated the hydrological variables of the correspondingly altered DEMs as well as their TEE and VarLEE values. It emerges that MoRE solutions feature far more optimal values of TEE and VarLEE than those randomly generated, confirming the suitability of Borg MOEA compared to a random choice of the parameters.

Finally, MoRE evaluates local morphological and hydrological conditions, namely Eq. (9), through the use of TopoToolbox software [Schwanghart and Kuhn, 2010]. The methodology embedded in the tool follows the classical approach: (i) the DEM is scanned in order to search for pits, which are sequentially filled; (ii) drainage directions and upslope areas are then evaluated through the D8 algorithm [O'Callaghan and Mark, 1984], and (iii) slope values along drainage lines are computed for each DEM cell. This procedure is repeated whenever a new DEM is generated as a candidate solution of the optimization problem.

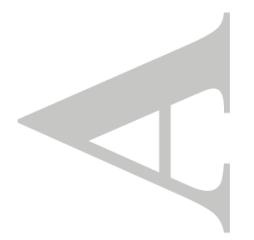

(C)2018 American Geophysical Union. All Rights Reserved. 


\section{Results}

\subsection{Structural Link between TEE and LEE}

We first investigate the link between TEE and VarLEE to understand which changes are necessary in the local energy distribution (VarLEE) to enhance channel network efficiency (TEE), or vice versa. Top row of Figure 1 shows the initial DEM A1 generated from a pyramid with flat surfaces by a random perturbation with Gaussian noise of cell elevations (see Methods). The Figure shows the 3D DEM, the resulting flow routing paths (shown as values of upslope area) and slope patterns.

Slope patterns clearly show a random patterns in elevation, with no emergence of any river profile or any other coherent basin morphology. However, the model creates cell elevation variations which guarantee that the flow from each cell reaches the pyramid boundaries and then create outlets. The generated flow paths create a network topology which cannot be easily evaluated at eyesight.

From the initial DEM A1, MORE generated a pool of 580 DEMs, represented by the blue dots filling the TEE-VarLEE space in Figure 2. MORE successfully searches for DEMs with smaller TEE and VarLEE values. Dots aligned along the curve between points $\mathrm{B}$ and $\mathrm{C}$ are Pareto efficient under the allowed altitude variation limit, i.e., they represent the best trade-offs between minimum TEE and minimum VarLEE within a constrained alteration of the initial pyramidal DEM ( \pm 20 units). The bent shape of the Pareto front implies that the system is forced to increase VarLEE in order to minimize TEE. In other words, DEM cells with the same upslope area (or equivalently discharge) are associated with a wider range of slopes, in order to accommodate more efficient river networks (smaller TEE). Two end member DEMs (points B and C) possess distinctive

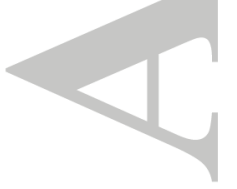

(C)2018 American Geophysical Union. All Rights Reserved. 
features: DEM B (lowest value of TEE) tends to have greater basins formed while DEM C (lowest value of VarLEE) has multiple disconnected smaller sub-basins in the same domain.

We simulate the evolution of possible channel network configurations through sequential experiments, each starting from an optimal DEM generated in the previous experiment. Any solution from the previous experiment can be a seed DEM for the next experiment. Here, we present a sequence of three consecutive experiments where A2 and A3 are selected as the seed DEMs for the second and third experiments, and MORE generates an additional 517 and 761 DEMs, respectively for these two additional experiments (red dots and yellow dots in Figure 2). In Figure 1 we can observe the striking difference in slope pattern emerging between DEM A1 characterized by the highest value of TEE (see Figure

2) and DEM A4 characterized by lowest values of TEE amongst all the DEM generated. Slope patterns in DEM A4 creates river profiles and morphologies where higher slopes (orange and red cells) drain into main channels represented by gentler slope (light and dark green cells) flowing from the top to the bases of the pyramid in multiples sub-basins.

The same difference in DEM organization is not appreciable at eyesight looking at the flow paths.

To better understand the changes occurred, we zoom in the major basin generated in the pyramid and observe more closely the trajectory of elevation changes and associated flow paths from DEM A1 to DEM A4. As can be observed in Figure 3 the main elevations changes occur in the first experiment (from A1 to A2), where main channels are carved and lateral sub-basins connected in order to flow into the main channel. As experiments are repeated, small-scale features are developed, such as minor incisions that increase the (C)2018 American Geophysical Union. All Rights Reserved. 
basin size by hooking small lateral basins in the upstream areas and in the lower part close to the pyramid boundary. Although we set the same constraint of \pm 20 units on local altitude variations for all experiments, elevation changes in the last two experiments decrease. This is coherent with what is suggested by Figure 2, where the Pareto-fronts get closer as optimization experiments follow one another. This implies that further changes in channel network efficiency to decrease TEE reduces as sequential experiments proceed, indicating a local optimum for the entire landscape.

Two main findings emerge from these experiments: i) VarLEE must increase to decrease TEE within a constrained alteration of the initial pyramidal DEM; ii) Increasing network efficiency through lowering TEE creates slope patterns which resembles morphological basin organization of real river network. In the next section we test if these morphological and topological features emerging from on-DEM OCN are statistically comparable to those observed in real river networks.

\subsection{Dual Necessities to Capture Both Network Self-Similarity and Channel}

\section{Profile Concavity}

Self-similarity in river networks can be presumed as described by power-law relationships among hydrogeomorphological variables [Tarboton et al., 1989; Rodriguez-Iturbe et al., 1992a; Rigon et al., 1996; Dodds and Rothman, 2000]. Among these topographical signatures, the probability distribution of the contributing area has been reported in the literature to follow a power-law relationship across basins of different size [Rodriguez-Iturbe et al., 1992a]. In a basin, the exceedance probability for a cell to have an upslope area $A$ greater than or equal to a random value $\delta$ is given by the power functional relationship

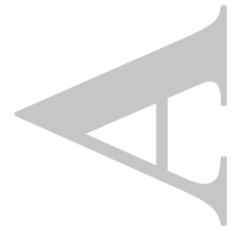

$$
P[A \geq \delta]=\delta^{-\varepsilon} F(\delta),
$$


where the exponent $\varepsilon$ is typically within a narrow range of $0.44 \pm 0.03$ [Rodriguez-Iturbe et al., 1992a]. Here, $F(\cdot)$ is a cutoff function to filter boundary effects [Maritan et al., 1996]. Another signature is found in the scaling of upstream length. The exceedance probability for a cell to have an upstream length $L$ greater than or equal to a random value $l$ is also represented as a power function [Rigon et al., 1996, see eq. (31) therein]:

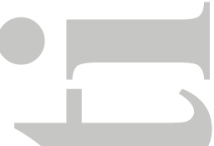

$$
P[L>l]=l^{-\gamma} F(l)
$$

where $F(\cdot)$ is the cutoff function. The exponent $\gamma$ in this relation is found to fall in the range $0.7-0.9$ [Rigon et al., 1996].

We adopt these power-laws to evaluate the self-similarity of networks obtained via MoRE. We evaluate the area exponent (Eq. (10)) and the $\gamma$ exponent (Eq. (11)) including all the cells of the major basins over the pyramid DEM, and considering up to four basins per DEM. The starting DEM A1 and the DEMs generated throughout the first experiment follow Eq. (10) and (11), as indicated by color intensity in Figure 4 (top and middle scatter plots). This may explain why these networks do not differentiate significantly at eyesight (see for instance upslope area graphs of DEM A1 and DEM A4 in Figure 1). However, their $\varepsilon$ are higher than the reported range of $0.44 \pm 0.03$ and rather close to 0.5 . This reflects the characteristic of a random binary tree [Paik and Kumar, 2007]. Similarly, $\gamma$ values exceed the range observed in nature at 0.9 . As the sequence of MoRE experiments proceeds resulting in lower and lower values of TEE, $\varepsilon$ and $\gamma$ values progress toward the range found in nature. This is consistent with earlier OCN studies [Paik, 2011].

Results from earlier studies suggest that the minimum TEE objective by itself does not generate concave longitudinal channel profiles [Paik, 2011, 2012]. Instead, the dual criteria of minimum TEE and VarLEE are necessary for profile concavity to emerge. (C)2018 American Geophysical Union. All Rights Reserved. 
Bottom graph of Figure 4 plots the exponent $\theta$ of Eq. (2). To calculate the slope-area relationship in each basin, we take the average slope for all cells having the same drainage area, following the procedure adopted in earlier studies [Tarboton et al., 1989; RodriguezIturbe et al., 1992a, details are shown in Figure 5]. Starting with nearly no concavity of the initial DEM A1, the trajectory of DEM features created in sequential applications of MoRE clearly follows the trend toward greater concavity as indicated by the increase of the fitted $\theta$ value. Further, the concavity relationship becomes robust as sequential simulations proceed: the coefficient of determination $\mathrm{R}^{2}$ of the slope-area relationship increases as TEE decreases. The concavity index $\theta$ varies widely in nature [Tarboton et al., 1991]. It has been suggested that $\theta$ values vary between bedrock and alluvial channels, and also depend on bed material size in alluvial channels, leading to a wide range of $0.3 ; \theta ; 1$ [Byun and Paik, 2017]. Because the landscape lithology is beyond the scope of current OCN theory, direct comparison of simulated $\theta$ with the values reported in nature is impractical.

Our results show that the slope-area relationship becomes relatively robust for DEMs with TEE values (normalized with respect to $\psi$ ) lower than $3.12 \times 10^{4}$ (see bottom plot of Figure 4). Interestingly, a similar TEE threshold can be claimed to identify DEMs with $\varepsilon$ and $\gamma$ values in the range reported for real river networks (see other plots of Figure 4). Hence, modeled channel networks with TEE values of $3.12 \times 10^{4}$ or lower possess topological (area exponent and the scaling of upstream length, top and middle row of Figure 4) and topographic (concavity index, bottom row of Figure 4) features resembling those observed in nature. These features emerge simultaneously for similar energy distributions, and suggest the existence of a structural link between network topology and topography

(C)2018 American Geophysical Union. All Rights Reserved. 
as the result of self-organizing processes emerging at the whole riverscape scale. Note that the threshold value of TEE identified above is only valid for our theoretical domain in this study, and the absolute threshold value depends on the size of the riverscape, and therefore on the total available potential energy and the initial configuration that binds the accessible, sub-optimal, and final networks.

\section{Discussion and Conclusions}

In this work, we reproduced realistic on-DEM riverscape topographies, using for the first time the OCN approach without a-priori imposing a slope-area relationship. We developed a Multi-objective Riverscape Exploration modeling framework, MoRE, which generates channel networks on DEMs pursuing the three principles of (i) minimum energy expenditure in any link, (ii) equal energy expenditure, and (iii) minimum total energy expenditure in the network as a whole. We embedded these principles in the formulation of the two objectives of minimum Total Energy Expenditure, TEE, and equal Local Energy Expenditure, VarLEE.

Unlike single-objective approaches [Rodriguez-Iturbe et al., 1992a; Paik, 2011], MoRE discloses multiple alternative DEMs, whose configurations best try to satisfy these two formulations of OCN principles. A key step in MoRE is the adoption of a function that expresses the elevation variation in each DEM cell in terms of local slope and drained area. This is an important advance compared to optimization routines based on random variations of the decision variable proposed by previous OCN literature [Paik, 2011; Rinaldo et al., 2014]. This function is implemented as a Radial Basis Function, whose parameters are optimized via the Borg Multi-Objective Evolutionary Algorithm to minimize the TEE and VarLEE OCN criteria.

(C)2018 American Geophysical Union. All Rights Reserved. 
Within a single optimization, under specific constraints of cell elevation alteration imposed to all riverscape cells (here \pm 20 elevation units), we demonstrated the trade-offs between the two criteria, i.e., the variance in LEE needs to increase in order to lower TEE. This fact was already found in the original OCN theory where networks (from random to optimal) during the simulating annealing process minimize the total energy and maximize the total entropy, which leads to maximize VarLEE [Rodriguez-Iturbe, I. and Rinaldo, A. , 2001].

When a specific degree of TEE is reached (about $3.12 \times 10^{4}$ for our simulation settings), MoRE generates multiple equally realistic networks. Indeed, they possess contributing area, stream length, and slope-area power-law relationships comparable to those observed in nature. When analyzing sequential experiments, and specifically those DEMs with similar VarLEE, yet decreasing TEE (e.g., DEMs A1, A2, and A3 in Figure 2), we observed OCNs with features comparable to those of real river networks. Indeed, most channel network configurations generated in the 2nd and 3rd sequential optimizations (orange and yellow dots in Figure 2) show topographical and topological features resembling those observed in nature (see Figure 4) independently from VarLEE. These evidences indicate a certain degree of sub-optimality in natural river networks. In nature, this sub-optimality can be imposed by different geologic or climatic settings. In an alternative perspective, there is a tendency in nature to settle down in easily reachable sub-optimal configurations [Rinaldo et al., 2014].

The role of the equal energy expenditure principle (highlighted by the objective of minimum VarLEE) is key in concavity formation. In each of our experiments, as already observed, we found that it is impossible to decrease both VarLEE and TEE (see the (C)2018 American Geophysical Union. All Rights Reserved. 
Pareto fronts in Figure 2). However, looking at the ensemble of all experiments, we can also observe that DEMs A1, A2, and A3 possess similar VarLEE values, but significantly different TEE (Figure 2) and concavity (bottom plot of Figure 4). Then, to further investigate the relations between TEE, LEE and concavity in Figure 5 we report a detailed comparison between DEMs A1 and A3. DEM A3 is characterized by a lower TEE, and the expected (mean) values of slope (darker and larger blue dots in Figure 5) scale with drained area generating robust river concavity. This is not happening in DEM A1, which is characterized by higher TEE. These findings point out the existence of a complex structural link between the scale-invariant properties found in river networks with minimum TEE, and the basin morphology required for an equal distribution of local energy expenditure (minimum VarLEE), in terms of slope-area relationship and river profile concavity. This structural link, which we have found to imply a trade-off among the two objectives, does not contradict the OCN principles. What we observe is that VarLEE is not uniformly equalized for decreasing TEE. Yet, we found that the expected value of local energy expenditure (LEE), calculated for cells having the same drained area, is constant throughout the basin. This statistical fact was discussed in the original OCN theory [Tarboton et al., 1991; Rinaldo et al., 1992]: the patterns of energy distribution are defined by power-law relationships between slope and area that embeds the multiscaling character of the energy expenditure processes, "whose expected value is the same throughout the network with a variance that increases proportionally to the average travel time for the flow to reach any site in the system". Indeed, slope variance increases in DEM A3 for smaller drained area (see smaller light blue dots in Figure 5), characterized by an higher travel time. Conversely, expected (mean) values (darker (C)2018 American Geophysical Union. All Rights Reserved. 
and larger blue dots in Figure 5) robustly comply with the slope-area relationship. In so generated on-DEM OCNs, slope-area relationship is not imposed a-priori. On the contrary, it emerges as a system feature that allows to pursue higher network efficiency, and guarantees the statistical validity of the OCN principle of equal energy expenditure per unit area. This fact also justifies the use of TEE for generating on-DEM OCNs, since the derivation of this criterion requires to satisfy the same principle of equal local energy expenditure.

In an attempt to provide a physical interpretation of these results, we can refer to the notion of rivers as hydraulic entities. Channel networks must develop to redistribute moisture, and sediment transport is a byproduct of hydrological processes [Phillips, 2010]. Soil moisture redistribution occurs through hydraulic selection principles: channels develop through concentrations of water and associated shear stress. These physical processes are at the basis of the tendency in nature towards building hydraulically efficient channel networks. This tendency is mediated by climate forcing, tectonic activity, geological, and lithological contexts. These context-specific parameters define how moisture redistribution and then channel developments take place [Phillips, 2007]. We postulate that these parameters uniquely define the accessible trade-off between the necessities to reduce the energy expenditure as a whole in the system and to equalize energy expenditure across the system. Different distribution of LEE at the landscape scale are likely to be the result of local climatic and geologic conditions. For instance, the values assumed by the concavity index in the 2nd and 3rd iterations of our experiments (see bottom plot of Figure 4) between -0.25 and -0.4 are likely affected by the particular setting of our artificial experiment. At this stage of an artificial experiment there is no mean to attempt 
to interpret these values in terms of basin processes and physical mechanism [Sinha and Parker, 1996]. However, they do generate concave profile comparable to those observed in real river network. Without embedding context-specific parameters, MoRE simulations showed the existence of equally realistic channel networks above a specific level of network organization. We can hypothesize that real river systems which have highly disorganized river networks, i.e., those with high values of TEE, are likely to be very unstable and quickly change configuration.

At present, the results we obtained by MoRE's search of alternative DEM configurations from an artificial, pyramidal DEM, do not allow us to argue whether any of the equally realistic OCN configurations we found is more likely than the others, or whether some act as an attractor states. To address this question we plan to generate alternative channel network configurations via MoRE starting from real-world DEMs, and to investigate how they position along the TEE-VarLEE trade-off. The increasing availability of high-resolution DEMs and computing power opens relevant opportunities to extend the scope of MoRE analyses up to the global scale. These experiments will help clarify and quantify the causal linkages between the tendency of river networks towards optimal network connectivity (TEE), the energy distribution across the basin (LEE), the physical processes acting in the basin, and its topological and topographic features.

\section{Acknowledgements}

We thank Patrice Carbonneau for his helpful comments on an early draft. We thank Patrick M. Reed for his support and training to use the Borg MOEA software. We would also like to thank the Associate Editor Riccardo Rigon, John Pitlick and two other anonymous reviewers for their relevant and thorough reviews. This work was supported by

(C)2018 American Geophysical Union. All Rights Reserved. 
the National Research Foundation of Korea (NRF) grant funded by the Korea government (MSIT) (No. 2018R1A2B2005772). No new data were used in producing this manuscript.

\section{Author contributions statement}

S.B., A.Ca., A.Co., E.M., and K.P. designed the research; A.Co. and E.M. developed the MoRE model; S.B., A.Co., and E.M. analyzed the model output; S.B., A.Ca., K.P. wrote the paper. All authors reviewed the manuscript.

\section{Additional information}

The authors declare no conflict of interest.

\section{Notes}

1. Available at: https://github.com/SALib/SALib, Copyright 2016 Jon Herman et al.

\section{References}

Abed-Elmdoust, A., M.-A. Miri, and A. Singh, Reorganization of river networks under changing spatiotemporal precipitation patterns: An optimal channel network approach, Water Resources Research, 52(11), 8845-8860, 2016.

Brierley, G., K. Fryirs, C. Cullum, M. Tadaki, H. Q. Huang, and B. Blue, Reading the landscape: Integrating the theory and practice of geomorphology to develop place-based understandings of river systems, Progress in Physical Geography, 37(5), 601-621, doi: 10.1177/0309133313490007, 2013.

Byun, J., and K. Paik, Small profile concavity of a fine-bed alluvial channel, Geology, 45, 627-630, doi:10.1130/G38879.1, 2017.

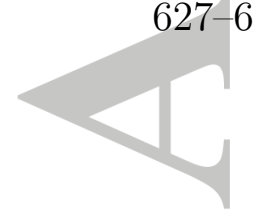

(C)2018 American Geophysical Union. All Rights Reserved. 
Coello, C. A. C., D. A. Van Veldhuizen, and G. B. Lamont, Evolutionary Algorithms for Solving Multi-Objective Problems, vol. 242, Springer, 2002.

Dodds, P. S., and D. H. Rothman, Scaling, universality, and geomorphology, Annual Review of Earth and Planetary Sciences, 28(1), 571-610, doi: 10.1146/annurev.earth.28.1.571, 2000.

Flint, J., Stream gradient as a function of order, magnitude, and discharge, Water Resources Research, 10, 969-973, 1974.

Hack, J. T., Interpretation of erosional topography in humid temperate regions, American Journal of Science, 258-A, 80-97, 1960.

Hadka, D., and P. Reed, Diagnostic assessment of search controls and failure modes in many-objective evolutionary optimization, Evolutionary Computation, 20(3), 423-452, 2012.

Hadka, D., and P. Reed, Borg: An Auto-Adaptive Many-Objective Evolutionary Computing Framework, Evolutionary Computation, 21(2), 231-259, 2013.

Horton, R., Erosional development of streams and their drainage basins; hydrophysical approach to quantitative morphology, Geological Society of America Bulletin, 56, 275370, 1945.

Kirby, E., and K. X. Whipple, Expression of active tectonics in erosional landscapes, Journal of Structural Geology, 44, 54-75, doi:10.1016/j.jsg.2012.07.009, 2012.

Kirkby, M., Hillslope process-response models based on the continuity equation, Institute of British Geographers Special Publication, 3, 15-30, 1971.

Leopold, L. B., and T. J. Maddock, The hydraulic geometry of stream channels and some physiographic implications, U. S. Geological Survey Professional Paper, 252, 1953.

(C)2018 American Geophysical Union. All Rights Reserved. 
Maritan, A., A. Rinaldo, R. Rigon, A. Giacometti, and I. Rodríguez-Iturbe, Scaling laws for river networks, Physical Review E, 53, 1510-1515, doi:10.1103/PhysRevE.53.1510, 1996.

O'Callaghan, J. F., and D. M. Mark, The extraction of drainage networks from digital elevation data, Computer Vision, Graphics, and Image Processing, 28(3), 323-344, 1984.

Paik, K., Optimization approach for 4-D natural landscape evolution, IEEE Transactions on Evolutionary Computation, 15(5), 684-691, doi:10.1109/TEVC.2010.2087027, 2011.

Paik, K., Search for the optimality signature of river network development, Physical Review E, 86(4), 046,110, doi:10.1103/PhysRevE.86.046110, 2012.

Paik, K., and P. Kumar, Inevitable self-similar topology of binary trees and their diverse hierarchical density, The European Physical Journal B, 60(2), 247-258, doi: 10.1140/epjb/e2007-00332-y, 2007.

Paik, K., and P. Kumar, Optimality approaches to describe characteristic fluvial patterns on landscapes, Philosophical Transactions of the Royal Society of London B: Biological Sciences, 365(1545), 1387-1395, doi:10.1098/rstb.2009.0303, 2010.

Park, J., and I. W. Sandberg, Universal approximation using radial-basis-function networks, Neural Computation, 3(2), 246-257, 1991.

Phillips, J. D., The perfect landscape, Geomorphology, 84(3), 159-169, 2007.

Phillips, J. D., The job of the river, Earth Surface Processes and Landforms, 35(3), 305$313,2010$.

Rigon, R., I. Rodriguez-Iturbe, A. Maritan, A. Giacometti, D. Tarboton, and A. Rinaldo, On Hack's law, Water Resources Research, 32(11), 3367-3374, 1996.

(C)2018 American Geophysical Union. All Rights Reserved. 
Rinaldo, A., I. Rodriguez-Iturbe, R. Rigon, R. L. Bras, E. Ijjasz-Vasquez, and A. Marani, Minimum energy and fractal structures of drainage networks, Water Resources Research, 28(9), 2183-2195, doi:10.1029/92WR00801, 1992.

Rinaldo, A., A. Maritan, F. Colaiori, A. Flammini, R. Rigon, I. Rodriguez-Iturbe, and J. R. Banavar, Thermodynamics of fractal networks, Physical Review Letters, 76, 33643367, doi:10.1103/PhysRevLett.76.3364, 1996.

Rinaldo, A., R. Rigon, and I. Rodriguez-Iturbe, Channel networks, Annual Review of Earth and Planetary Sciences, 26, 289-327, 1998.

Rinaldo, A., R. Rigon, J. R. Banavar, A. Maritan, and I. Rodriguez-Iturbe, Evolution and selection of river networks: Statics, dynamics, and complexity, Proceedings of the National Academy of Sciences of the United States of America, 111(7), 2417-24, doi: 10.1073/pnas.1322700111, 2014.

Rodriguez-Iturbe, I., E. Ijjasz-Vasquez, R. L. Bras, and D. Tarboton, Power law distributions of discharge mass and energy in river basins, Water Resources Research, 28(4), 1089-1093, 1992a.

Rodriguez-Iturbe, I., A. Rinaldo, R. Rigon, R. L. Bras, A. Marani, and E. Ijjasz-Vasquez, Energy dissipation, runoff production, and the three-dimensional structure of river basins, Water Resources Research, 28(4), 1095-1103, 1992b.

Rodriguez-Iturbe, I. and Rinaldo, A. , Fractal river basins chance and self organization: Hydrology, hydrogeology and water resources, August 27, 2001 revised ed., Cambridge University Press, 2001.

Saltelli, A., P. Annoni, I. Azzini, F. Campolongo, M. Ratto, and S. Tarantola, Variance based sensitivity analysis of model output. design and estimator for the total sensitivity (C)2018 American Geophysical Union. All Rights Reserved. 
index, Computer Physics Communications, 181(2), 259-270, 2010.

Schumm, S. A., Evolution of drainage systems and slopes in badlands at Perth Amboy, New Jersey, Geological Society of America Bulletin, 67(5), 597-646, doi:10.1130/00167606(1956)67[597:EODSAS]2.0.CO;2, 1956.

Schwanghart, W., and N. J. Kuhn, TopoToolbox: A set of Matlab functions for topographic analysis, Environmental Modelling \& Software, 25(6), 770-781, doi: 10.1016/j.envsoft.2009.12.002, 2010.

Sinclair, K., and R. C. Ball, Mechanism for global optimization of river networks from local erosion rules, Physical Review Letters, 76, 3360-3363, doi: 10.1103/PhysRevLett.76.3360, 1996.

Sinha, S. K., and G. Parker, Causes of concavity in longitudinal profiles of rivers, Water Resources, 32(5), 1417-1428, 1996.

Sun, T., P. Meakin, and T. Jøssang, Minimum energy dissipation model for river basin geometry, Physical Review E, 49, 4865-4872, doi:10.1103/PhysRevE.49.4865, 1994.

Tarboton, D. G., R. L. Bras, and I. Rodriguez-Iturbe, Scaling and elevation in river networks, Water Resources Research, 25(9), 2037-2051, 1989.

Tarboton, D. G., R. L. Bras, and I. Rodriguez-Iturbe, On the extraction of channel networks from digital elevation data, Hydrological Processes, 5(1), 81-100, doi: 10.1002/hyp.3360050107, 1991.

Willgoose, G., A physical explanation for an observed area-slope-elevation relationship for catchments with declining relief, Water Resources Research, 30(2), 151-159, doi: 10.1029/93WR01810, 1994.

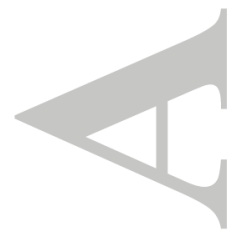

(C)2018 American Geophysical Union. All Rights Reserved. 
Woldenberg, M., Spatial order in fluvial systems: Horton's laws derived from mixed hexagonal hierarchies of drainage basin areas, Geological Society of America Bulletin, 80, $97-112,1969$.
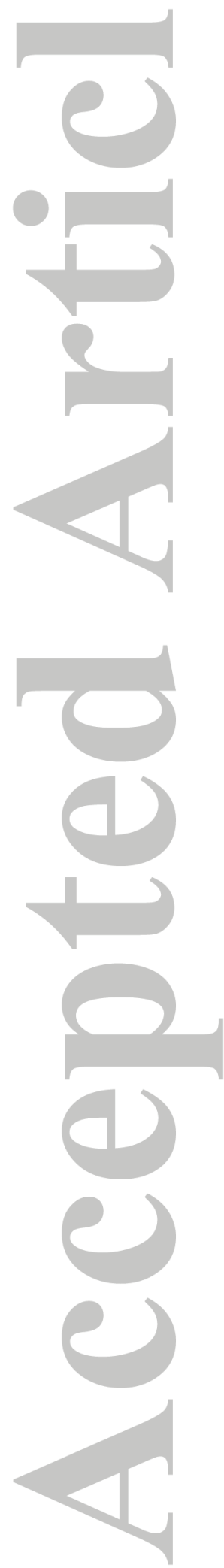

(C)2018 American Geophysical Union. All Rights Reserved. 


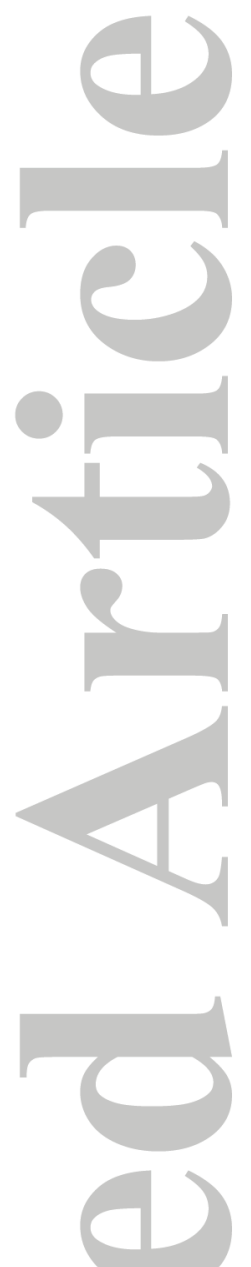

Figure 1. 3D DEM, spatial distribution of upslope area, and local slope of the initial DEM

A1 (top) and of DEM A4 (bottom) are reported. To see where DEM A1 and A4 are located in the TEE-VarLEE space see Figure 2. In the upslope area figure the lines represent the flow paths: their color and width is based on the amount of drained cell. Slope is reported in terms of tangent of the steepest downward altitude gradient: slope pattern show that the carved river network developed on DEM A4 is remarkably different from the pristine landscape of DEM A1, although the networks may look similar at eyesight.

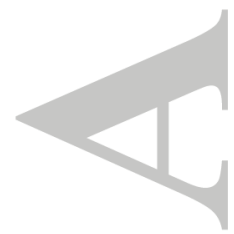

(C)2018 American Geophysical Union. All Rights Reserved. 

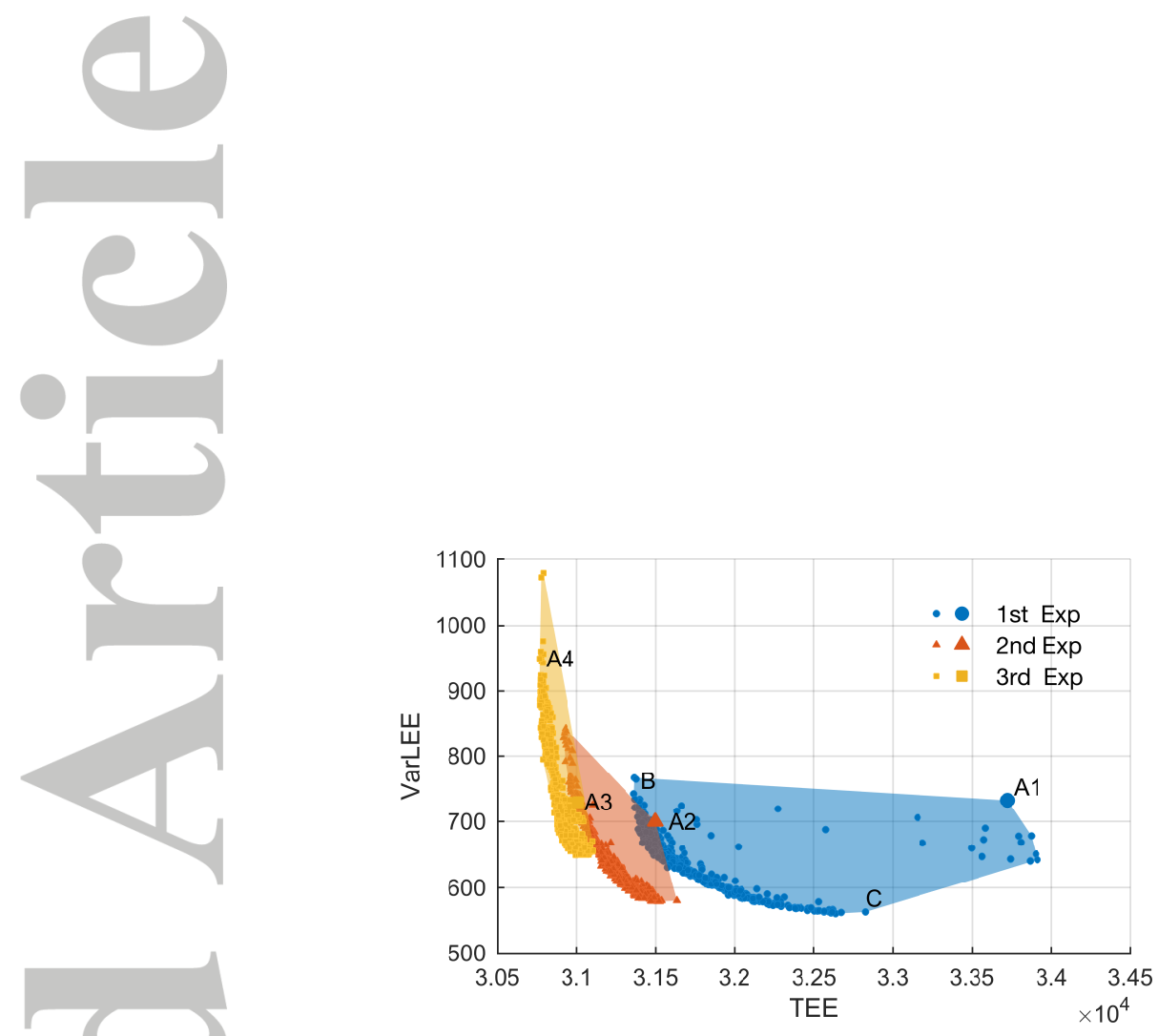

Figure 2. TEE-VarLEE space. Each dot represents a DEM generated by MoRE across three sequential experiments. Experiments are denoted by different colors, according to the legend. The starting DEM for experiment $i$ is denoted as $A i$ and represented with bigger size dots in the scatter plot. DEMs B and C are two extremes from the first experiment.

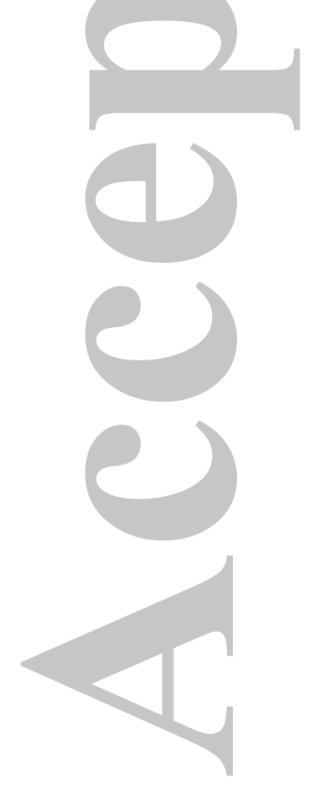

(C)2018 American Geophysical Union. All Rights Reserved. 


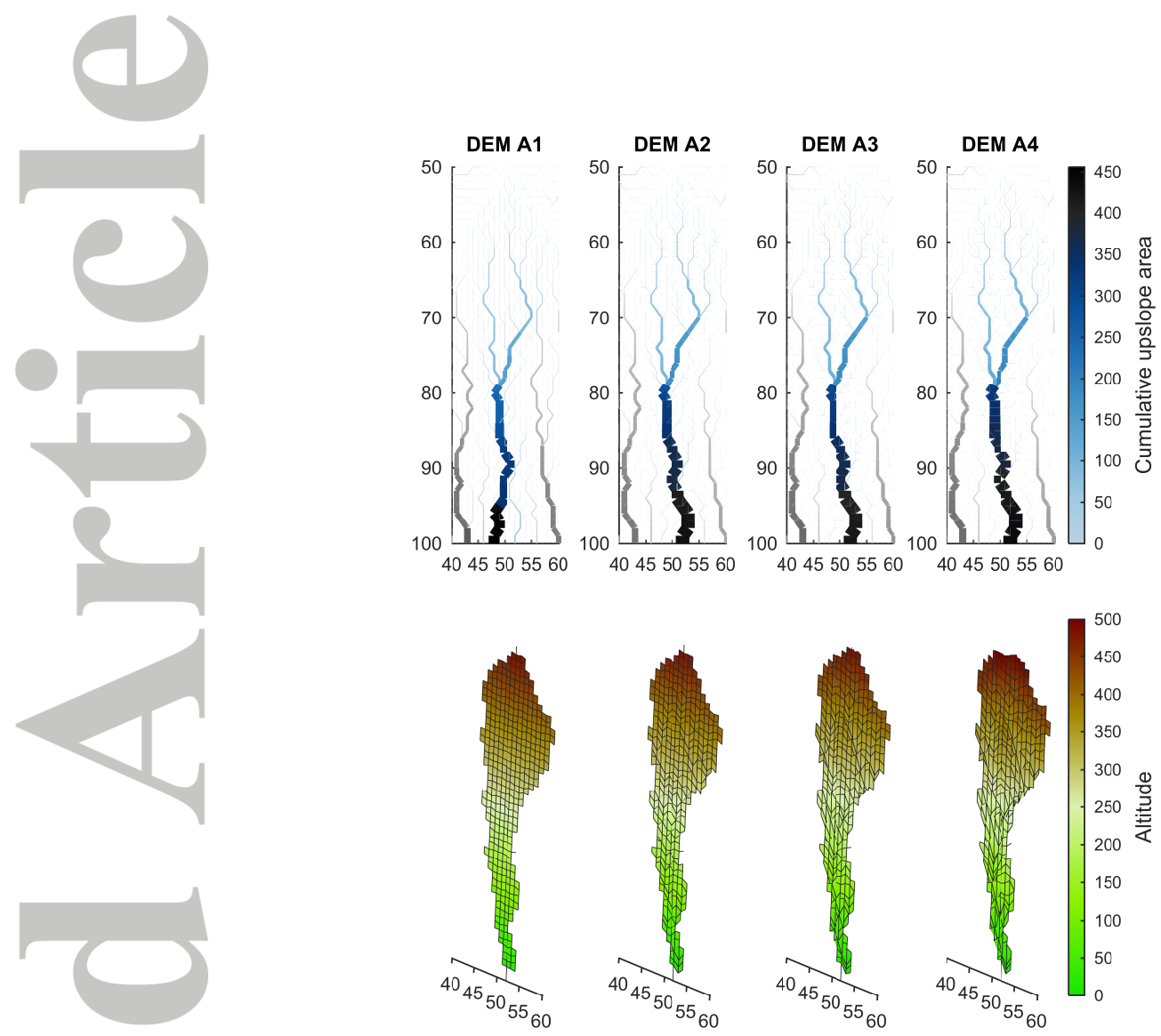

Figure 3. Development of river network configuration (top) and shaded relief (bottom) along sequential experiments with MoRE. DEM A1 is chosen as the initial DEM of the first experiment, followed by DEMs A2 and A3 as seeds of the second and third experiments, and A4 was chosen as representative final DEM after three experiments. Figures show the development for the portion of domain corresponding to the greatest basin formed in A4. Line thickness and color in top figures are proportional to the upslope area. Bottom plots show DEM morphology, where color is proportional to cell altitude.

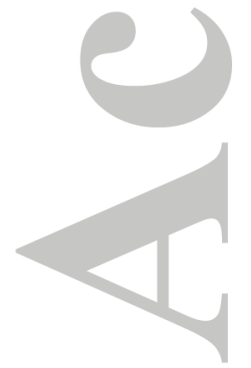

(C)2018 American Geophysical Union. All Rights Reserved. 

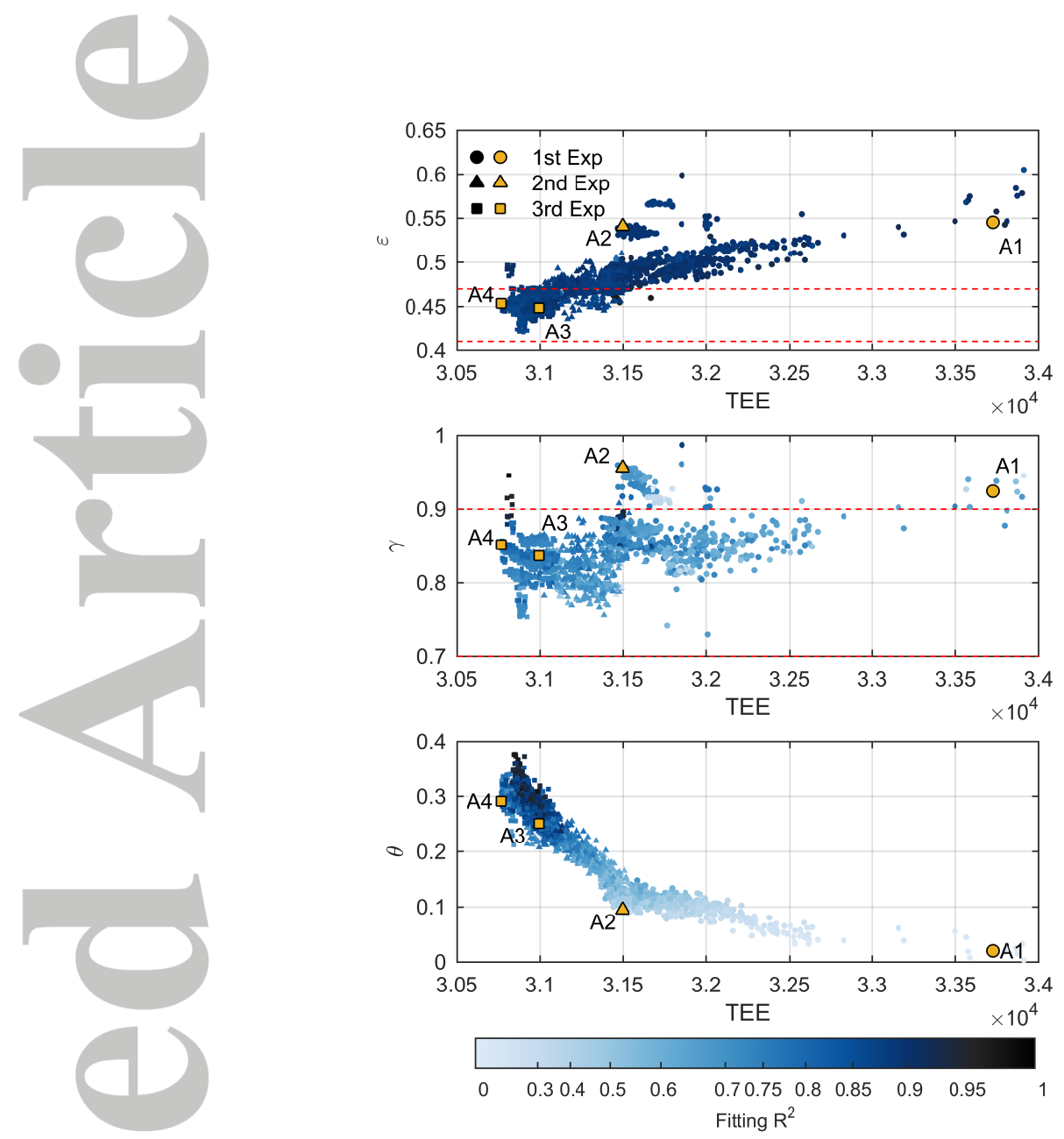

Figure 4. Exponents $\varepsilon$ of Eq. (10), on top, $\gamma$ of Eq. (11), in the middle, and $\theta$ of Eq. (2), on bottom, plotted versus TEE for all generated DEMs through the three sequential experiments from DEMs A1 (circles), A2 (triangles), and A3 (squares). The bigger yellow dots represent the starting DEMs of each experiment. Dot color is proportional to the coefficient of determination $R^{2}$. Horizontal red dashed lines in the top and middle charts bound the range of typical values of $\varepsilon$ and $\gamma$ suggested in the literature.

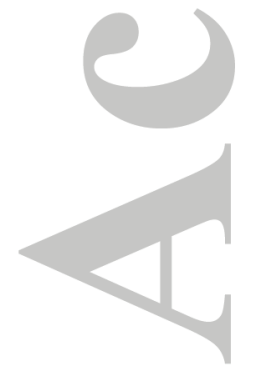

(C)2018 American Geophysical Union. All Rights Reserved. 

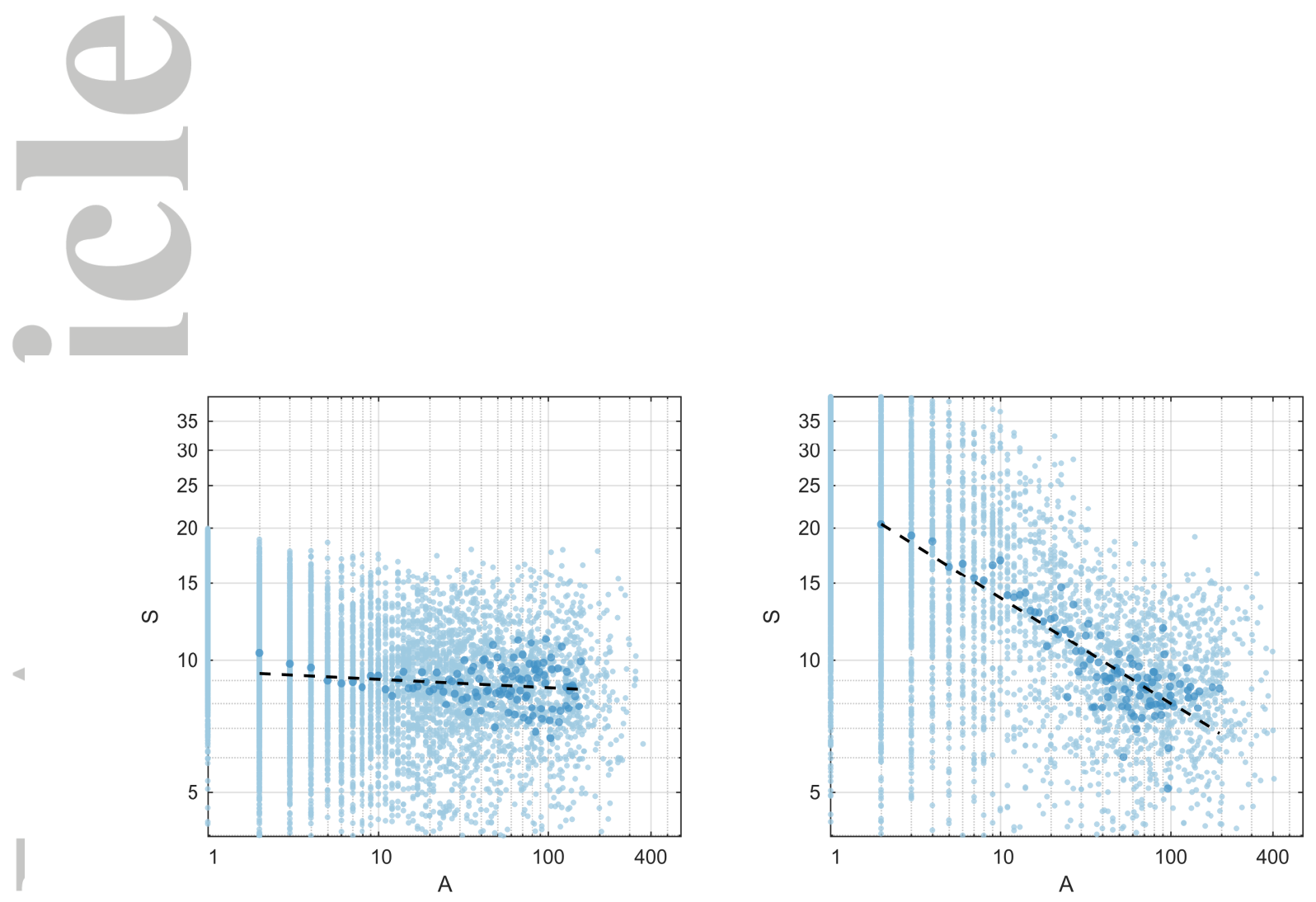

Figure 5. Slope-area relations for DEM A1 (left) and A3 (right) of Figure 2, both having similar VarLEE. Lighter blue dots indicate values for all cells of the pyramidal DEM, while darker blue dots are the mean slope values of cells with the same drained area. The dashed black line is the fitted slope-area relation for each DEM, in log space. 